$$
\text { تأثتير المحولات المؤرضة على دقة قياس مرحّلة حماية المسافة لخطوط النقل المزدوجة }
$$

يتضمن هذا البحث دراسة تأثير المحولة المؤرضة في خطوط النقل المزدوجة ذات التفرّع الوسطي تجهز

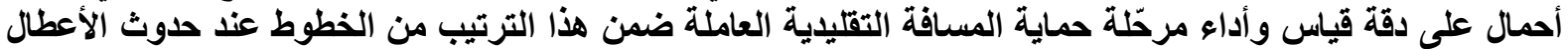

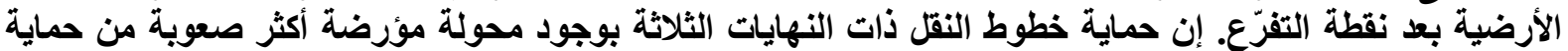

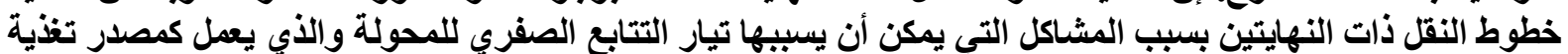

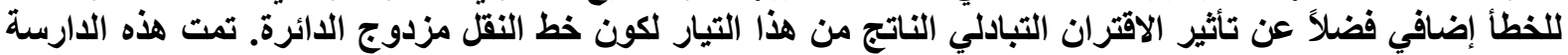

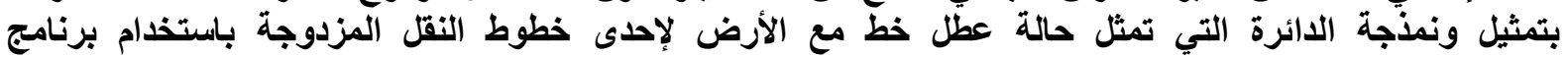

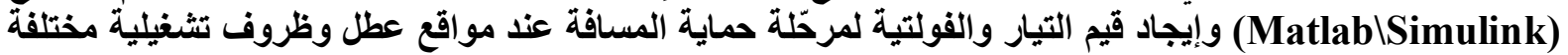

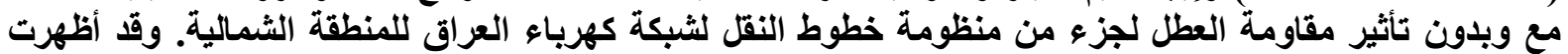

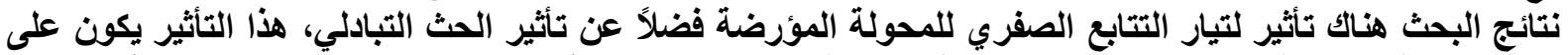

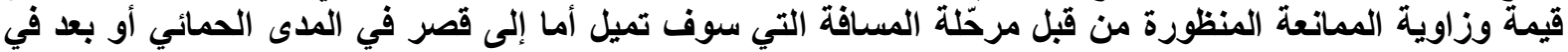

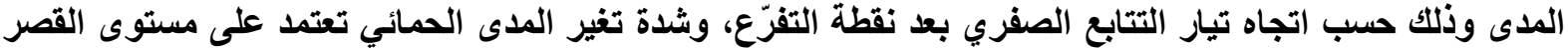

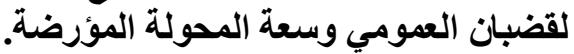

\title{
Influence Of an Earthed Transformers On The Distance Protection Relay Measuring Accuracy For Double Transmission Lines
}

\author{
Dr. Abdul Ghani A. Abdul \\ Ghafoor \\ Electrical Department \\ College of Engineering \\ University of Mosul
}

\author{
Mr. Ahmed Ateya Al- \\ Badrani \\ Electrical Department \\ Institute of Mosul \\ Foundation of Technical Education
}

\section{Abstract:}

This paper investigates a study of the influence of an earthed transformer in a double circuit transmission lines have intermediate tapped to provide to loads on a measuring accuracy and performance of the conventional Distance Protection Relay (DPR) installed in this configuration when the ground faults occur beyond tee point. The protection of three-terminal lines is not as simple as that of two-terminal lines. They usually experience problems caused by the zero sequence current in-feed of an earthed transformer from the third terminal as well as mutual coupling due to this current. This study has been modeling and simulating by using (Matlab $\backslash$ Simulink) program to analyze a steady state of ground fault on single circuit to calculate the DPR voltage, current and apparent impedance with various fault locations under operational status for the Iraqi North Regional Grids (INRG) $132 \mathrm{kV}$ system. The paper results shows that current in-feed and mutual coupling by zero sequence of earthed transformer may cause the distance relay to seriously under-reach or Under Reach according to zero sequence current direction. The values of short circuit levels ratio of system have major effect in this problem, So an apparent impedance as seen by DPR and length of protective zone will change.

Keywords: Distance Protection Relay, Earthed Transformer, Mutual Coupling Effect, Fault location. 
نظر أًًَ للأهمية البالغة التي تتمتع بها مرحّلات حماية المسافة في حماية خطوط النقل الكهربائية، فإن التقنيات

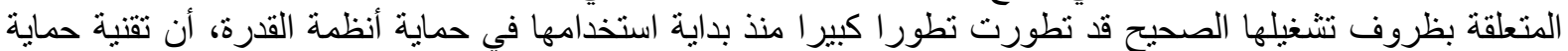

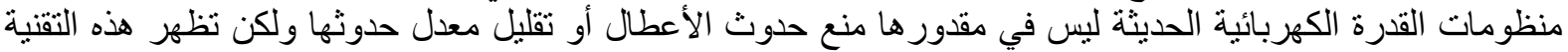
قدرتها في إمكانية تقصير الفترة الزمنية لتواجد انخفاض الجية الجهد في شبكة النقل من خلال تحسية تحسين الأداء و واستخدام حماية تتصف بدقة عالية وسرعة تثنغيل كبيرة جدًا. إن المعضلة الأساسية في الحماية المعتمدة على المئل المسافة تكمن في عدم التمكن

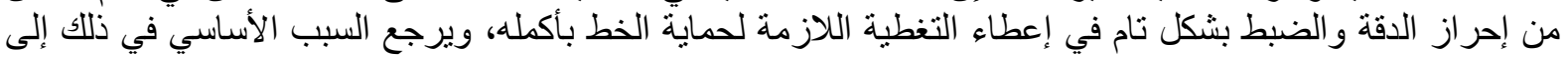

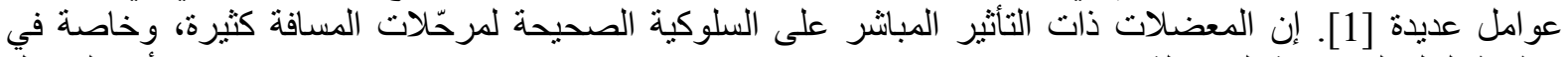
خطوط النقل المزدوجة المتداخلة (Interconnected Double Transmission Lines) [2] ، ويمكن ذكر أهم العوامل المؤثرة بالآتي:

\section{1. تأثير خط التفرّع الوسطي في خطوط النقل الكهربائية:}

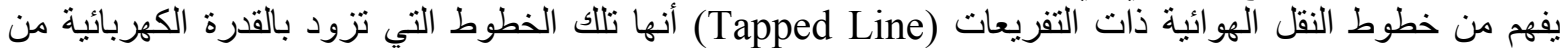

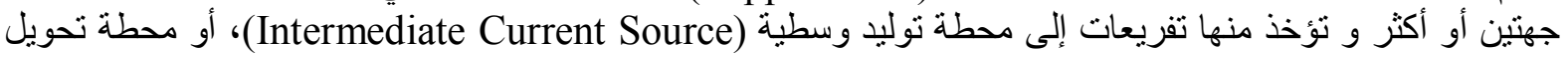

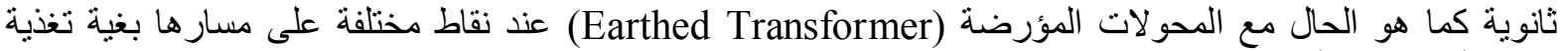

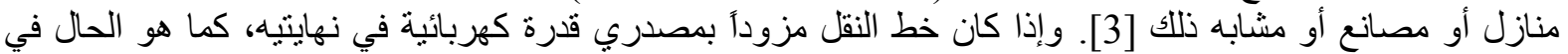

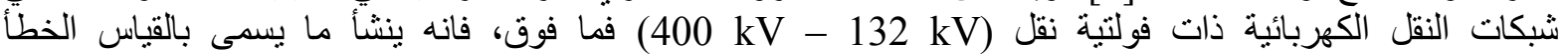

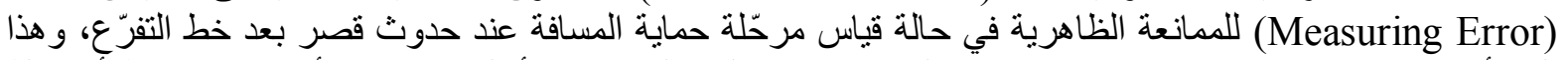

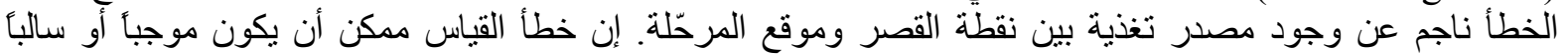

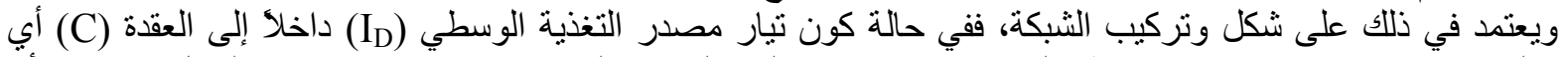

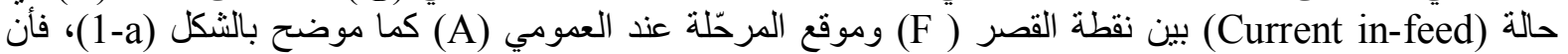

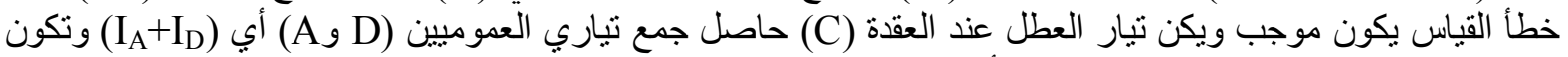

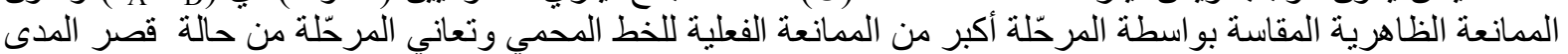

.[8][6](Under Reach)

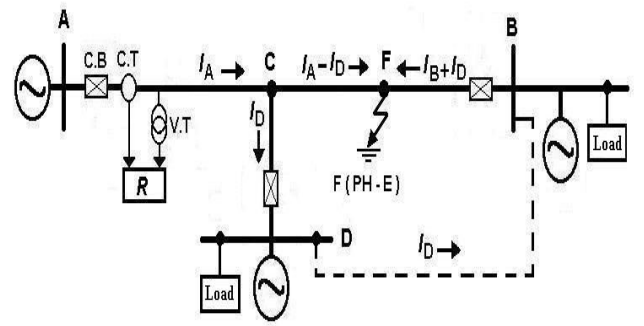

$-\mathrm{b}-$

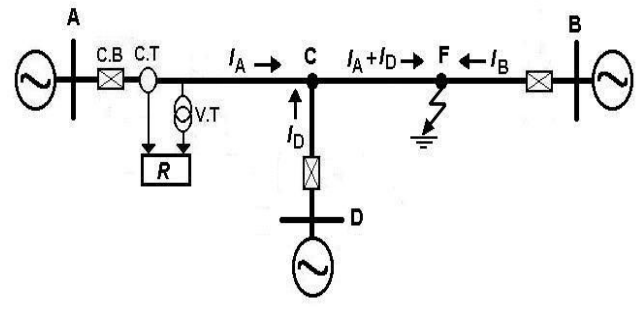

$-\mathrm{a}-$

$$
\text { الثكل (1): رسم توضيحي لخط نقل ذو ثلاثة نهايات بوجود عطل ارضي }
$$

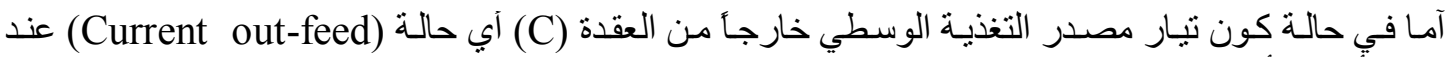

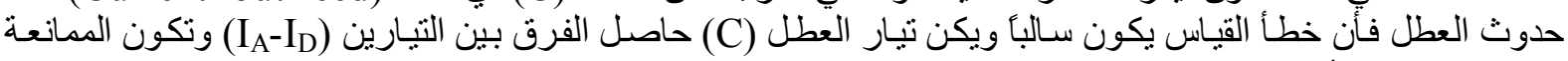

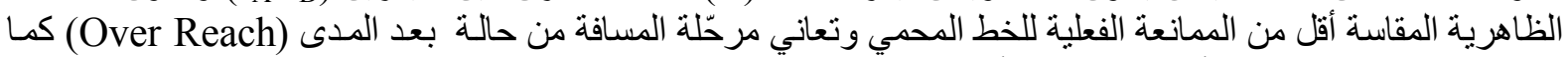

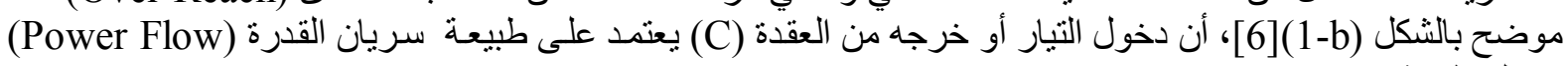
في المنظومة.

2. تأثير الحث التبادلي في خطوط النقل الهوائية المزدوجة الدائرة:

إن خطوط النقل الهو ائية ذات الدائرة المزدوجة (Double Circuit) على نفس البرج يحدث فيها اقتران حثي

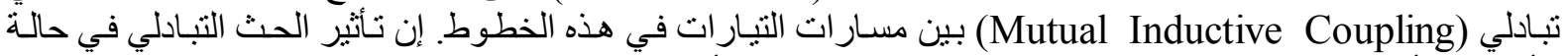

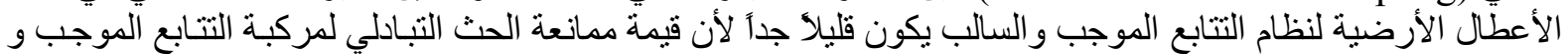

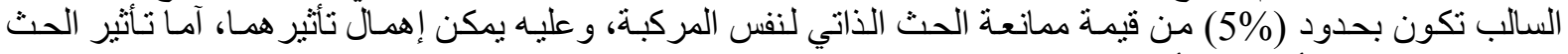

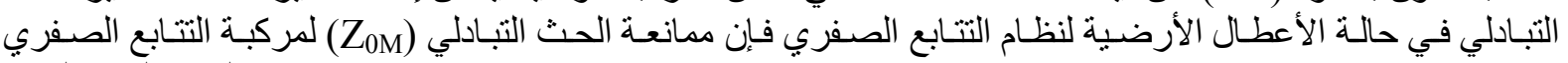
(Zero Sequence Mutual Impedance)

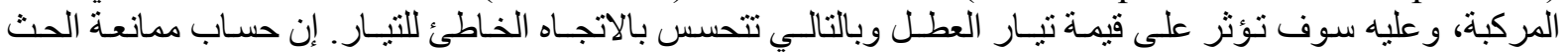


التبادلي بين نظامي التتابع الصفري بدون تأثير سلك التأريض المعتمد في هذا البحث يكون وفق الأسلوب المتبع لثـركة (5iemens)

$$
\mathrm{Z}_{0 \mathrm{M}}=3 \cdot \mathrm{R}_{\mathrm{E}}+\left(\mathrm{j} . \omega \cdot 6 \cdot \ln \frac{D_{e}}{A_{L}}\right) \cdot 10^{-4} \quad[\Omega / \mathrm{km}]
$$

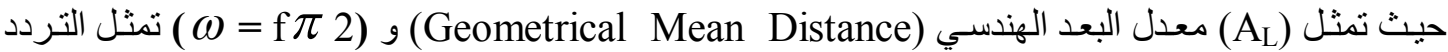

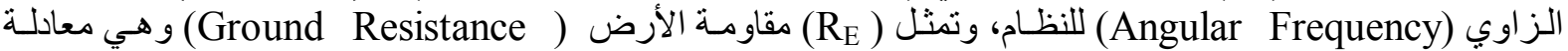

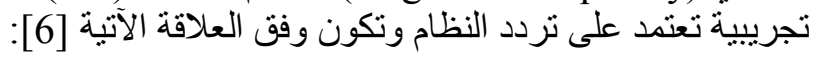

$$
\mathrm{R}_{\mathrm{E}}=(\pi / 2) \cdot \omega \cdot 10^{-4} \quad[\Omega / \mathrm{km}]
$$

آما ( Denetration Depth ( ) لمتمثل مدى النفوذ ) و الذي يمثل بدوره عمق الاختر اق المكافئ لمسار التيار

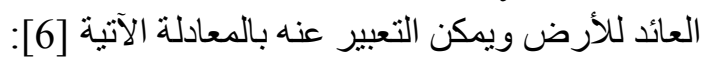

$$
D_{e}=1650 \sqrt{\frac{\rho}{m}}
$$

حيث إن ( م ) تمثل المقاومة النو عية للأرض (Specific Resistance of Earth)

3. ت تأثير مقاومة العطل:

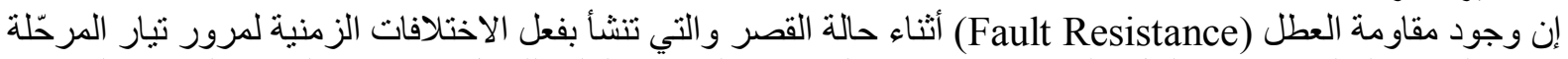

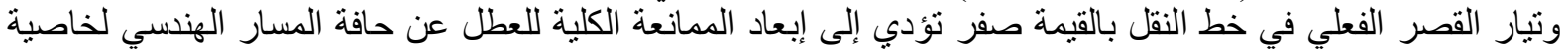

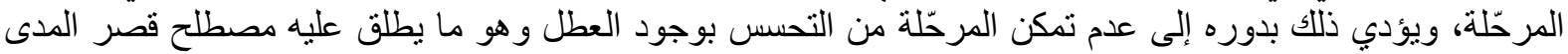

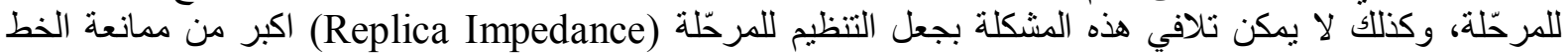

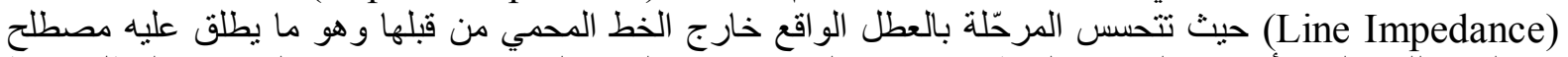

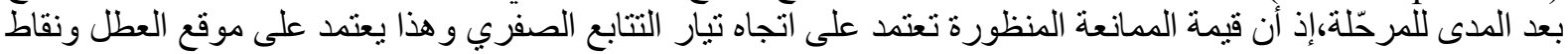

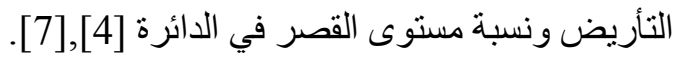

\section{ظروف تثثغيل مرحّلة قياس المسافة بوجود محولة مؤرضة في خط التفرّع:}

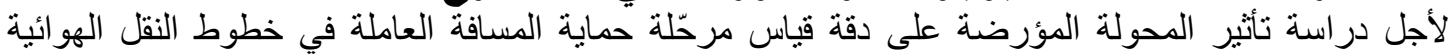
Iraqi North (المزدوجة لثبكة كهرباء العراق للمنطقة الثمالية (Over Head Transmission Lines OHTL) Regional Grids INRG ذات جها (132 kV)، نم اختيار جزء من هذه المنظومة وكما هو موضح في الشكل (2),

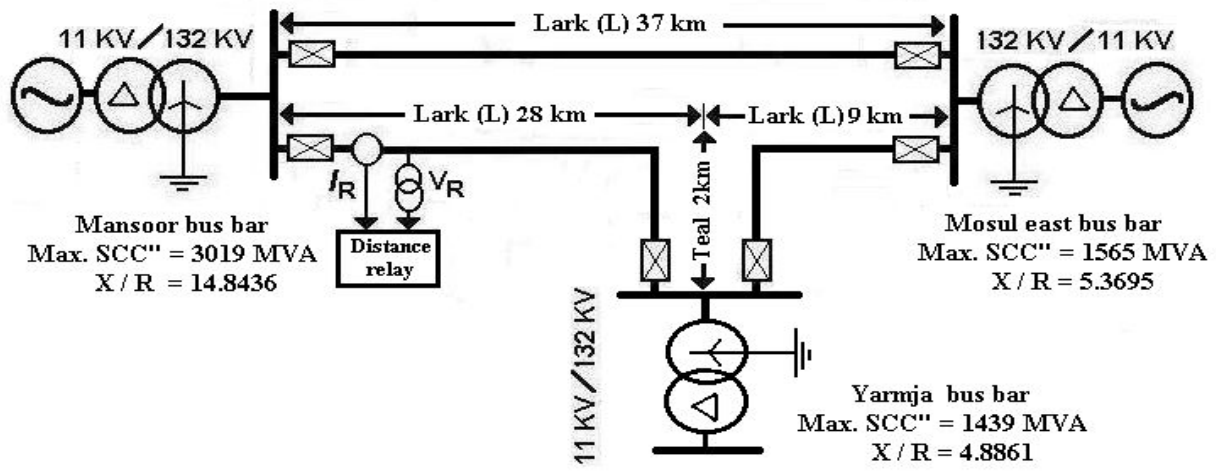

الشكل (2): جزء من خطوط النقل الهو ائية المزدوجة (132 kV) بوجود محولة مؤرضتفي خط تفرّع لثبكة كهرباء العر اق للمنطقة الثمالية 


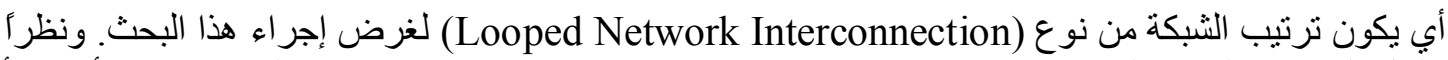

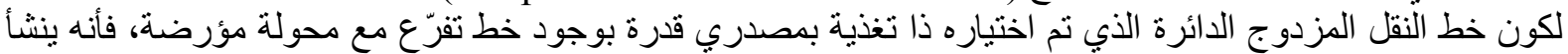

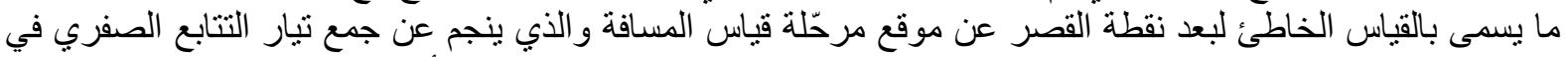

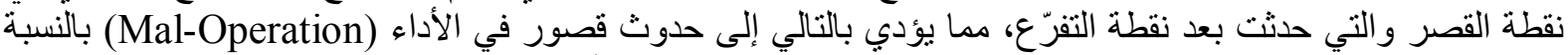

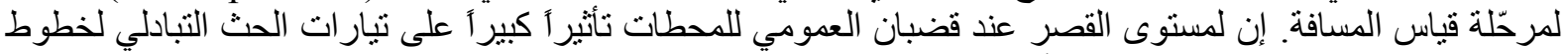

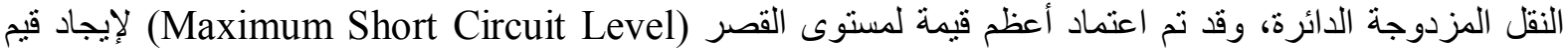
الممانعات المكافئة للمصادر عند قضبان العمومي المعنية وكما مبينة في الجدول (1).

الجدول (1) : قيم ممانعات التعاقب الموجبة ( أو السالبة ) و الصفرية للمصادر المكافئة

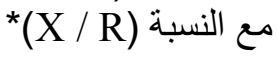

\begin{tabular}{|c|c|c|c|}
\hline $\mathrm{X} \backslash \mathrm{R}$ & $\mathrm{Z}_{0 \mathrm{~S}}[\Omega]$ & $\mathrm{Z}_{1 \mathrm{~S}}[\Omega]$ & قبضنان العمومي \\
\hline 14.843 & $2.073+\mathrm{j} 2.979$ & $0.383+\mathrm{j} 5.749$ & المنصور الغازية \\
\hline 5.369 & $4.931+\mathrm{j} 16.08$ & $2.038+\mathrm{j} 10.94$ & موصل الشرقية \\
\hline- & $5.035+\mathrm{j} 17.47$ & - & يارمجة \\
\hline
\end{tabular}

(*) ملاحظة: البيانات في الجدول أعلاه مأخوذة من الثركة العامة لإنتاج الطاقة الكهربائية للمنطقة

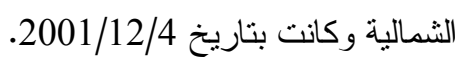

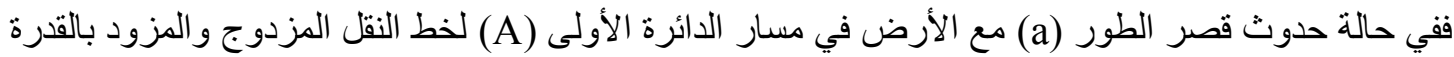

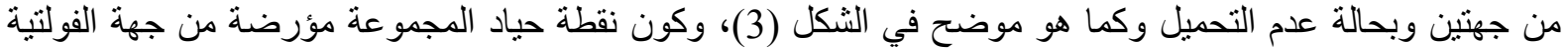

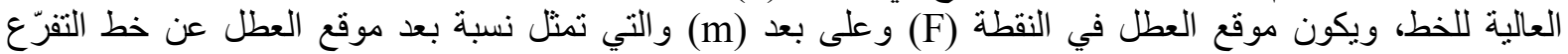

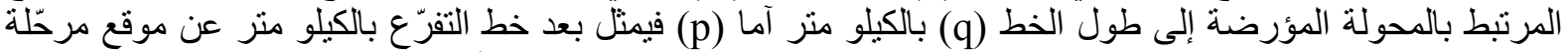

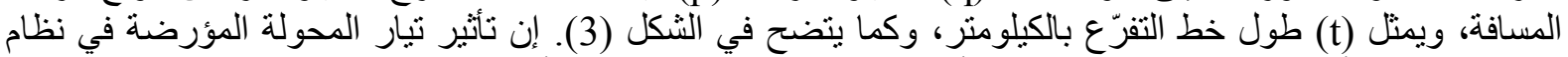

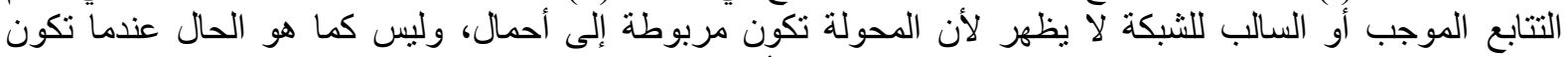

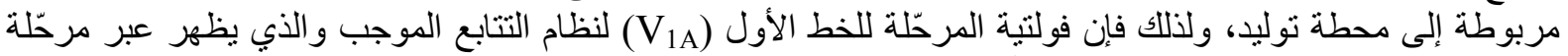

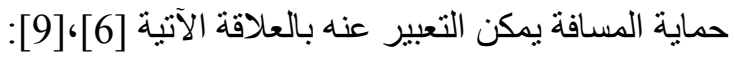

$$
\mathrm{V}_{1 \mathrm{~A}}=(\mathrm{p}+2 \mathrm{t}+\mathrm{m} \cdot \mathrm{q}) \cdot \mathrm{Z}_{1 \mathrm{~L}} \cdot I_{1 \mathrm{~A}}+\mathrm{V}_{1 \mathrm{~F}}
$$

وكذللك الحال بالنسبة لفولتية المرحّلة (V2A) نظام التتابع السالب للمرحّلة وكالآتي [6]:

$$
\mathrm{V}_{2 \mathrm{~A}}=(\mathrm{p}+2 \mathrm{t}+\mathrm{m} \cdot \mathrm{q}) \cdot \mathrm{Z}_{2 \mathrm{~L}} \cdot I_{2 \mathrm{~A}}+\mathrm{V}_{2 \mathrm{~F}}
$$

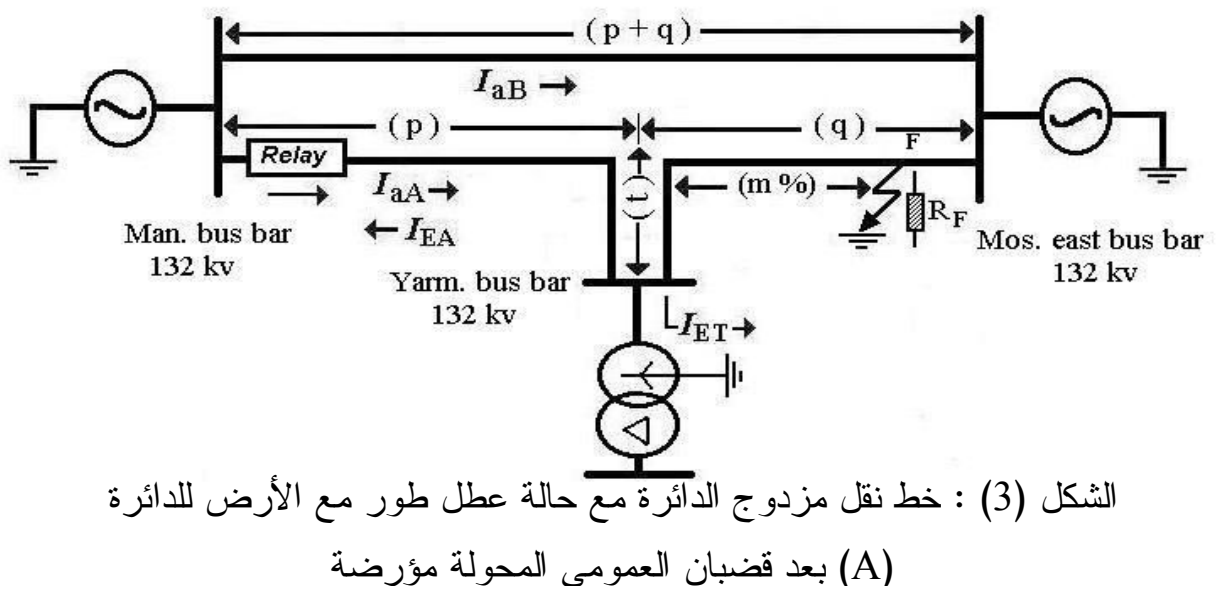


حيث أن تيار التتابع الموجب للمرحّلة يكون مساوياً لتبار التتابع السالب (

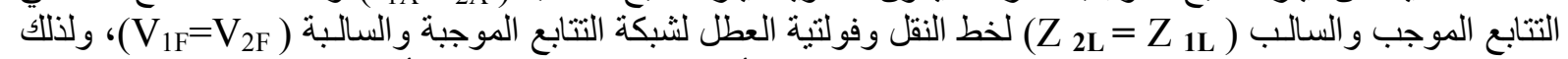

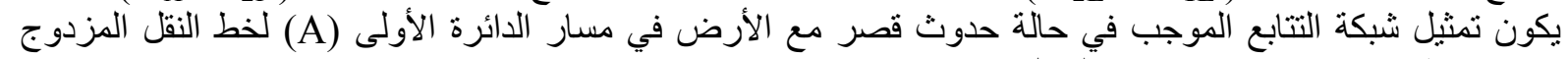

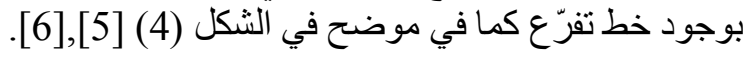

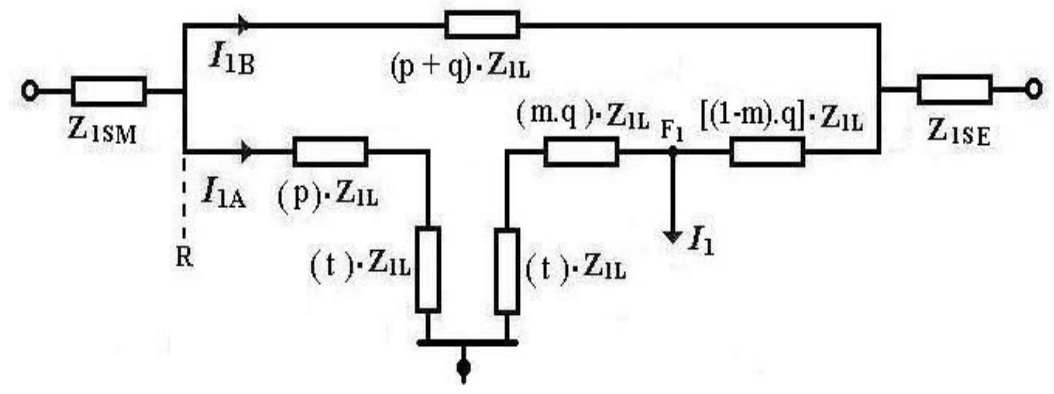

$$
\text { الشكل (4): شبكة التعاقب الموجب في حالة حدوث قصر مع الأرض في }
$$

\section{صفرية}

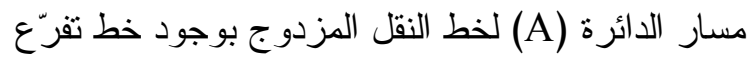

إذ أن

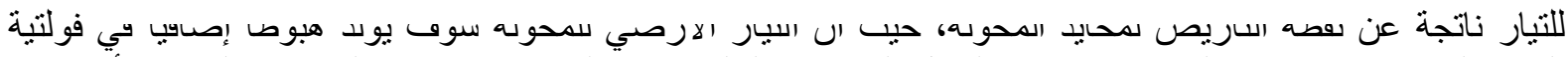

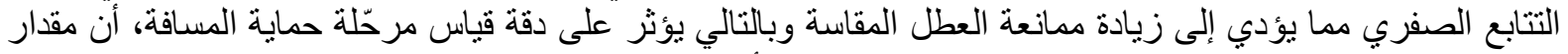

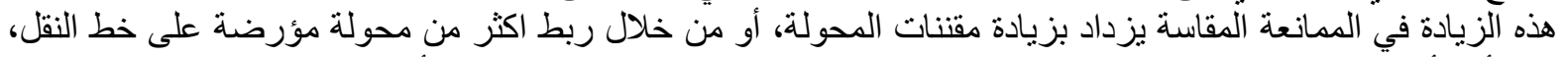

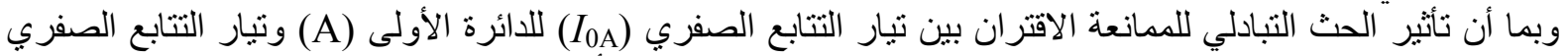

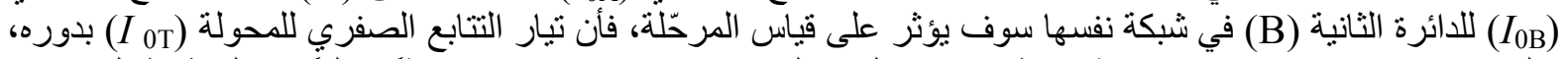

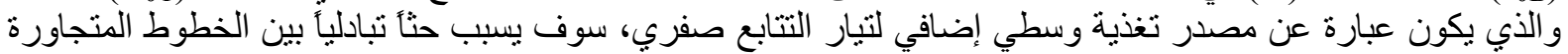

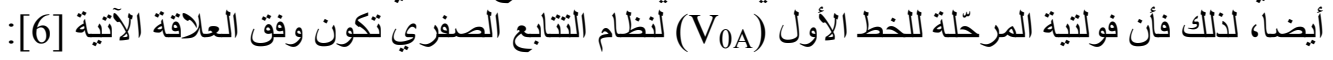

$$
\begin{gathered}
\mathrm{V}_{0 \mathrm{~A}}=\left[(\mathrm{p}+2 \mathrm{t}+\mathrm{m} \cdot \mathrm{q}) \cdot \mathrm{Z}_{0 \mathrm{~L}}-(2 \cdot \mathrm{t}) \cdot \mathrm{Z}_{0 \mathrm{M}}\right] \cdot I_{0 \mathrm{~A}}+\left[(\mathrm{p}+\mathrm{m} \cdot \mathrm{q}) \cdot \mathrm{Z}_{0 \mathrm{M}}\right] \cdot I_{0 \mathrm{~B}} \ldots \\
{\left[(\mathrm{t}+\mathrm{m} \cdot \mathrm{q}) \cdot \mathrm{Z}_{0 \mathrm{~L}}-(\mathrm{t}) \cdot \mathrm{Z}_{0 \mathrm{M}}\right] \cdot I_{0 \mathrm{~T}}+\mathrm{V}_{0 \mathrm{~F}}}
\end{gathered}
$$

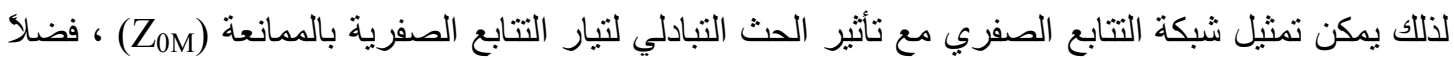

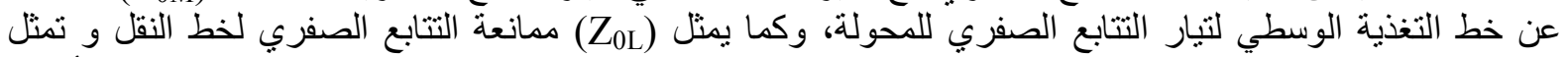

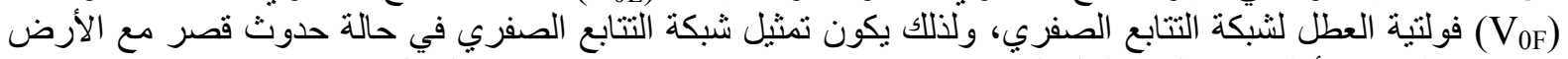

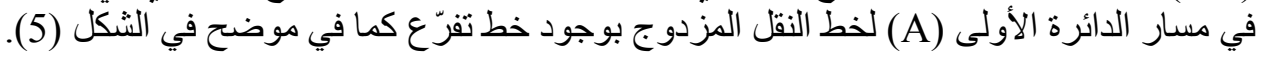

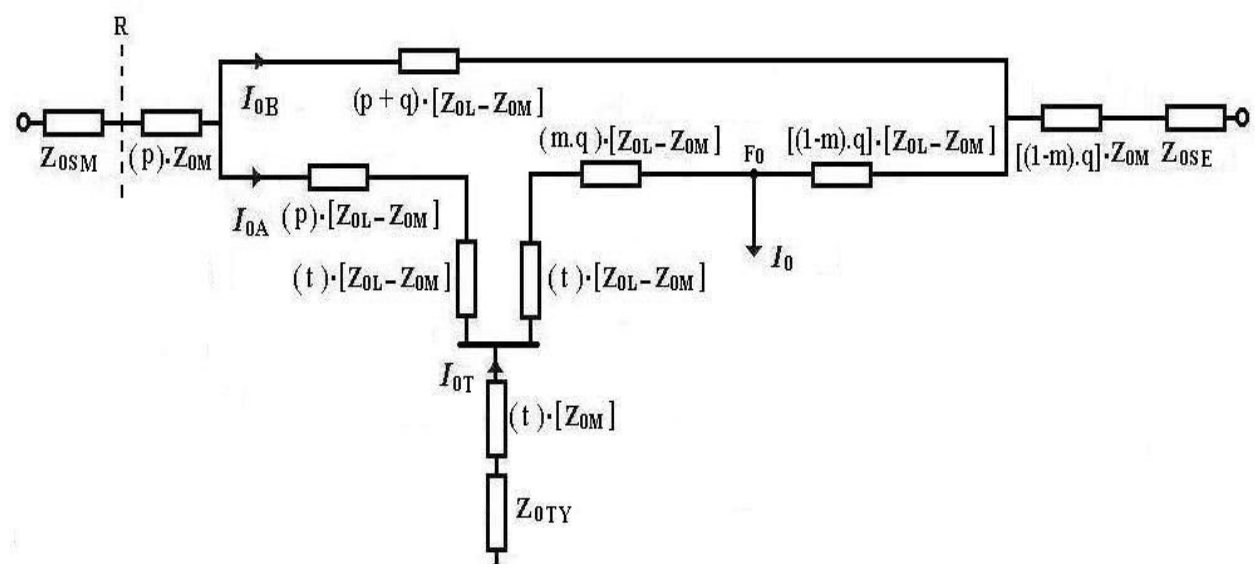

الثكل (5): شبكة التعاقب الصفري في حالة حدوث قصر طور مع الأرض

في مسار الدائرة (A) لخط النقل المزدوج بوجود خط تفرّع 
و عليه فانن الفولتية التي تظهر عبر مرحّلة قباس المسافة (VR) و التي تثمل هبوط الجهد لمركبات التتابع الثناثة

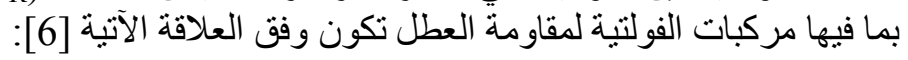

$\mathrm{V}_{\mathrm{R}}=\mathrm{V}_{100 \mathrm{~A}}+\mathrm{V}_{2 \mathrm{~A}}+\mathrm{V}_{0 \mathrm{~A}}+3 \cdot I_{\mathrm{F}} \cdot \mathrm{R}_{\mathrm{F}}$

وحيث يمثل (I) تيار العطل بينما تمثل (RF) مقاومة العطل (Fault Resistance) و التي تشمل كل من مقاومة

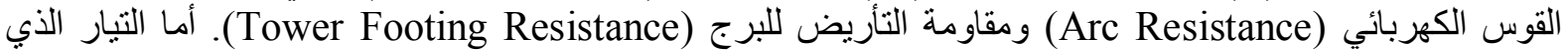

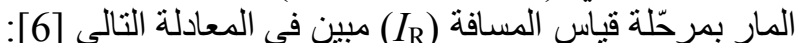

$$
I_{\mathrm{R}}=\left(I_{1 \mathrm{~A}}+I_{2 \mathrm{~A}}+I_{0 \mathrm{~A}}\right)+\left(\frac{Z_{0 L}-Z_{1 L}}{Z_{1 L}}\right) \cdot I_{0 \mathrm{~A}}
$$

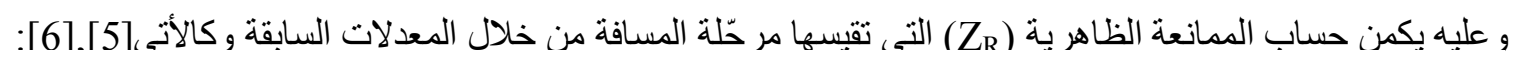

$$
\mathrm{Z}_{\mathrm{R}}=\frac{V_{R}}{I_{a A}+K_{E} \cdot I_{E A}}
$$

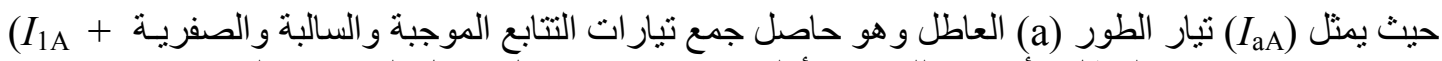

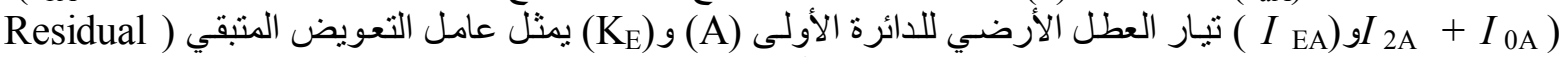
(6],[6] وكما في المعادلة الآتية: (Compensation Factor

$$
\mathrm{K}_{\mathrm{E}}=\frac{L_{0 L}-L_{1 L}}{3 Z_{1 L}}
$$

وقد تم استخدام برنامج (Matlab\Simulink + M-File) لتمثيل و نمذجة مرحّلة قياس المسافة العاملة في خطوط النقل الهوائية المزدوجة (132kV) لشبكة كهرباء العراق للمنطقة الثمالية من خلال تحليل حالة عطل أرضي

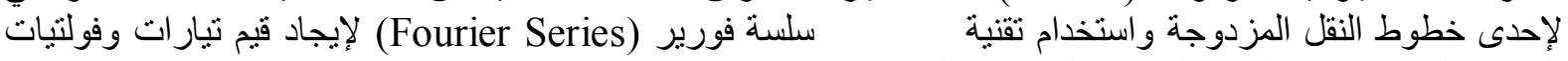
التتابع و المنظومة فضلا عن الممانعة الظاهرية المقاسة.

\section{النتائج و المناقشة:}

تم في هذه الدراسة بيان تأثير تبار التتابع الصفري للمحولة المؤرضة والئة الواقعة بين موقع مرحّلة حماية المسافة

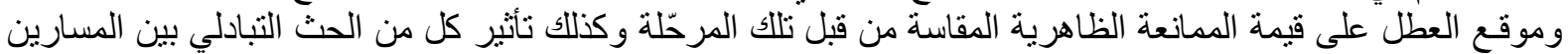

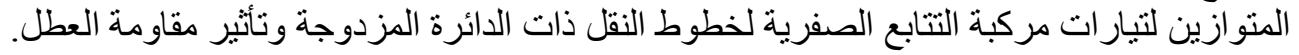

تأثثير المحولة المؤرضة في خط التقرّع على مرحّلة حماية المسافة:

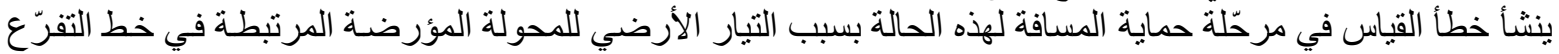

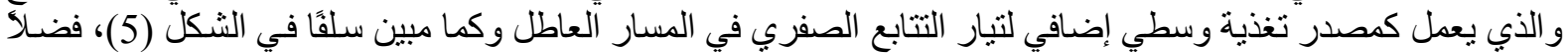

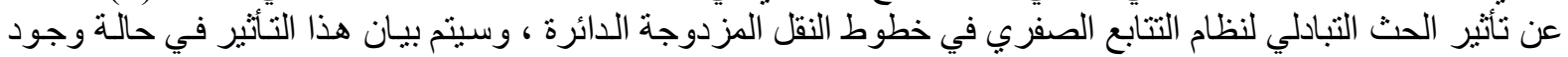

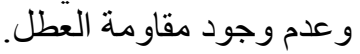

1. الة عدم وجود مقاومة العطل:

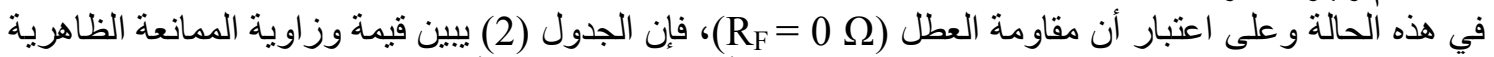

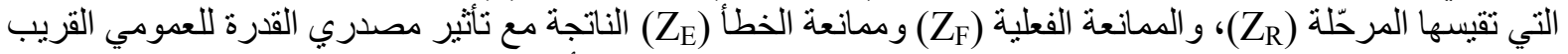

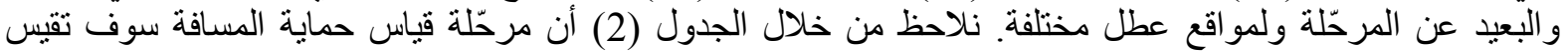

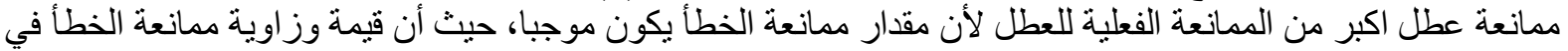

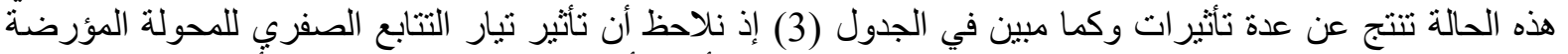
( مع تيار التتابع الصفري (I0T)

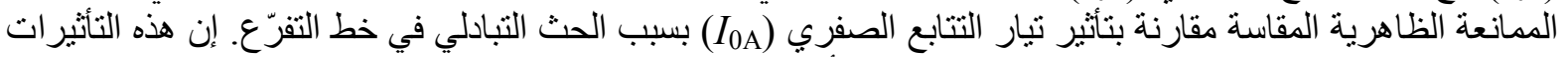

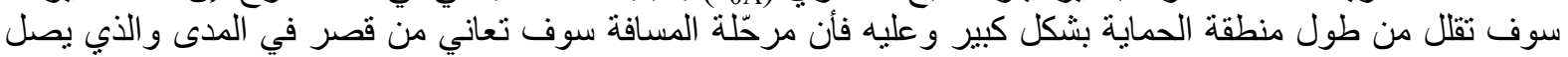

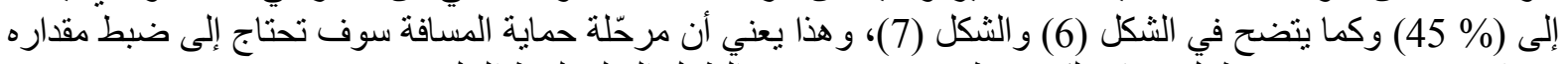
بمقدار (\% (181.5) من طولَ مساره لكي يغطي (6) ولئ (7) 100) من النطول الفعلي لخط النقل. 
عبد الففور: تأثير المحولات المؤرضة على دقة قياس مرحّلة حماية المسافة لخطوط النقل المزدوجة
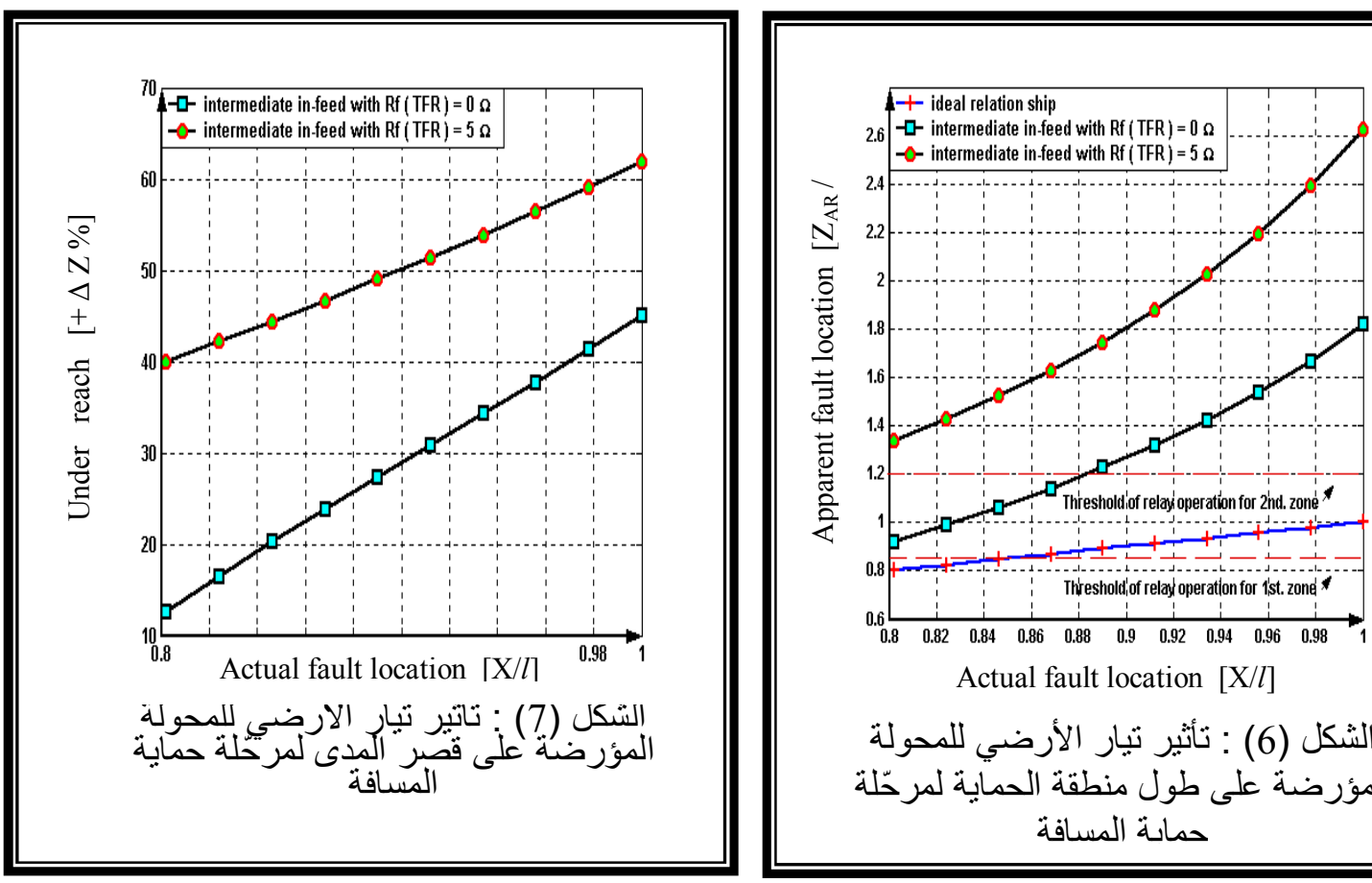

الشكل (6) : تأثير نيار الأرضي للمحولة

المؤرضة على طول منطقة الحماية لمرحّلة

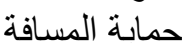

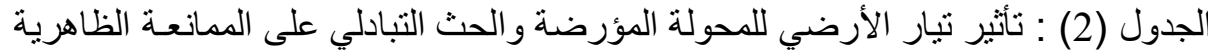

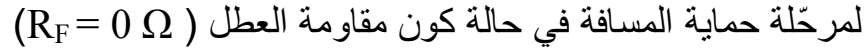

\begin{tabular}{|c|c|c|c|}
\hline 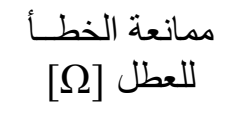 & 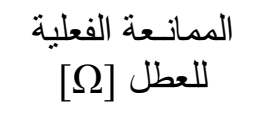 & اللمرحمّلة الظـاهرية & لموقع العطلة المئوية \\
\hline $73.7 \angle 02.845$ & $69.8 \angle 14.404$ & $70.4 \angle 17.244$ & $\mathrm{~m}=20 \%$ \\
\hline $73.8 \angle 04.740$ & $69.8 \angle 15.171$ & $70.7 \angle 19.902$ & $\mathrm{~m}=40 \%$ \\
\hline $74.1 \angle 07.092$ & $69.8 \angle 15.938$ & $71.1 \angle 23.017$ & $\mathrm{~m}=60 \%$ \\
\hline $74.2 \angle 10.134$ & $69.8 \angle 16.705$ & $71.4 \angle 26.902$ & $\mathrm{~m}=80 \%$ \\
\hline $74.4 \angle 10.304$ & $69.8 \angle 17.472$ & $71.9 \angle 31.751$ & $\mathrm{~m}=100 \%$ \\
\hline
\end{tabular}

الجدول (3) : قيم وزوايا ممانعات الخطأ بسبب التيار الأرضي للمحولة المؤرضة وممانعة الاقتران

التبادلي بسبب تيار ات التعاقب الصفرية في حالّة مقاومة عطل ( R

\begin{tabular}{|c|c|c|c|c|}
\hline 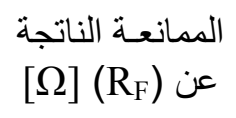 & 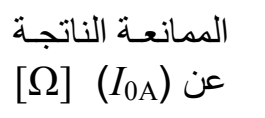 & 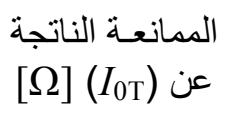 & 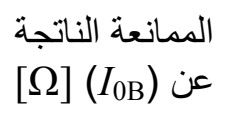 & لموقع العطلة المئو \\
\hline 0.00 & $-101.8<0.420$ & $68.3 \angle 1.321$ & $78.2 \angle 1.954$ & $\bar{~}$ \\
\hline 0.00 & $-101.9 \angle 0.401$ & $69.6 \angle 2.379$ & $77.1 \angle 2.775$ & $\mathrm{~m}=40 \%$ \\
\hline 0.00 & $-102.1 \angle 0.376$ & $70.3 \angle 3.602$ & $77.8 \angle 3.882$ & $\mathrm{~m}=60 \%$ \\
\hline 0.00 & $-102.2 \angle 0.342$ & $70.8 \angle 5.067$ & $77.7 \angle 5.427$ & $\bar{m}=80 \%$ \\
\hline 0.00 & $-1027 \angle 0.291$ & $71.1 \angle 6.916$ & $77.5 \angle 7.701$ & $\mathrm{~m}=100 \%$ \\
\hline
\end{tabular}




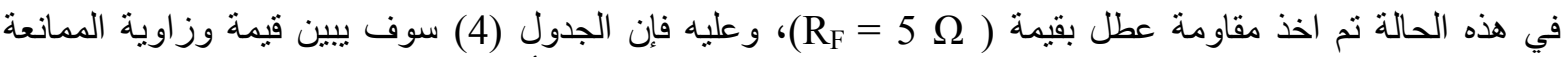

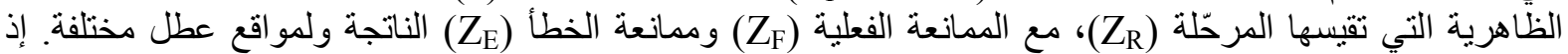

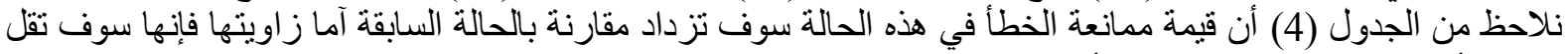

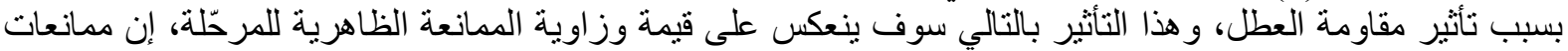

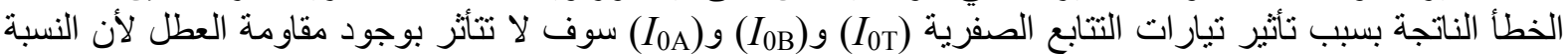

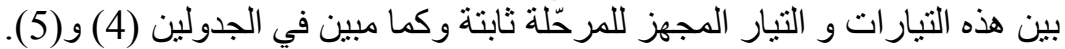

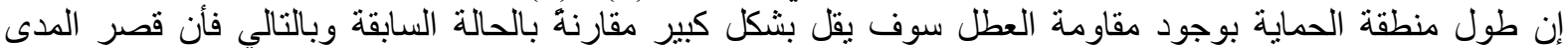

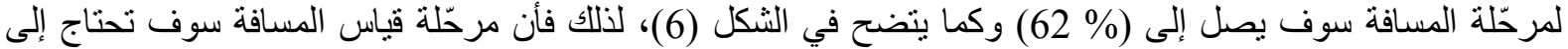
ضبط مقداره بمقدار (\% 262.5) من طول مسار لكي يغطي (62\% وكي (100) من الطول الفعلي لخط النقل.

الجدول (4) : تأثثر تبار الأرضي للمحولة المؤرضة والحث التبادلي على الممانعــة الظاهرية

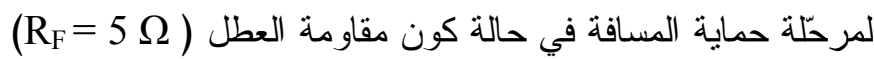

\begin{tabular}{|c|c|c|c|}
\hline 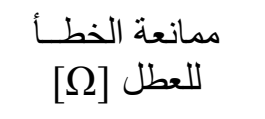 & 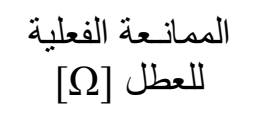 & 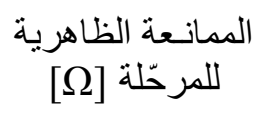 & لموقع العطلة المئوية \\
\hline $8.80 \angle 14.471$ & $69.8 \angle 14.404$ & $39.2 \angle 24.882$ & $\mathrm{~m}=20 \%$ \\
\hline $13.4 \angle 17.069$ & $69.8 \angle 15.171$ & $39.8 \angle 28.433$ & $\mathrm{~m}=40 \%$ \\
\hline $17.4 \angle 20.519$ & $69.8 \angle 15.938$ & $40.1 \angle 32.755$ & $\mathrm{~m}=60 \%$ \\
\hline $20.0 \angle 25.239$ & $69.8 \angle 16.705$ & $39.9 \angle 38.321$ & $\mathrm{~m}=80 \%$ \\
\hline $24.4 \angle 32.049$ & $69.8 \angle 17.472$ & $39.4 \angle 45.879$ & $\mathrm{~m}=100 \%$ \\
\hline
\end{tabular}

الجدول (5) : قيم ممانعات الخطأ بسبب التيار الأرضي للمحولة المؤرضة مع نيار التعاقب الصفري بسبب

\begin{tabular}{|c|c|c|c|c|}
\hline 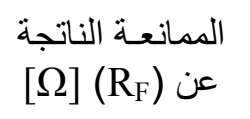 & 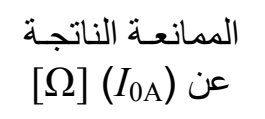 & 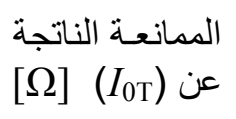 & 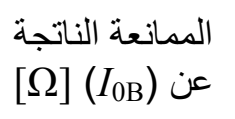 & لموقع العطلة المئوية \\
\hline$-2.1 \angle 13.508$ & $-101.8 \angle 0.420$ & $68.3 \angle 1.321$ & $78.2 \angle 1.954$ & $\mathrm{~m}=20 \%$ \\
\hline$-2.1 \angle 15.291$ & $-101.9 \angle 0.401$ & $69.6 \angle 2.379$ & $77.1 \angle 2.775$ & $\mathrm{~m}=40 \%$ \\
\hline$-2.1 \angle 17.642$ & $-102.1 \angle 0.376$ & $70.3 \angle 3.602$ & $77.8 \angle 3.882$ & $\mathrm{~m}=60 \%$ \\
\hline$-2.2 \angle 20.868$ & $-102.2 \angle 0.342$ & $70.8 \angle 5.067$ & $77.7 \angle 5.427$ & $\mathrm{~m}=80 \%$ \\
\hline$-2.3<25.588$ & $-1027 \angle 0.291$ & $71.1 \angle 6.916$ & $77.5 \angle 7.701$ & $\mathrm{~m}=100 \%$ \\
\hline
\end{tabular}

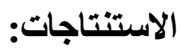

إن نتائج هذا البحث مشتقة من تحليل حالة عطل طور مع الأرض لخط نقل مزدورج الدائرة مزود بالقدرة من

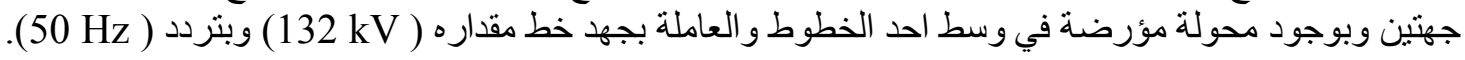

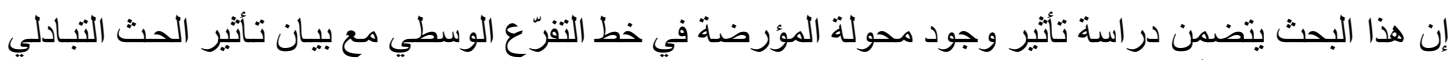

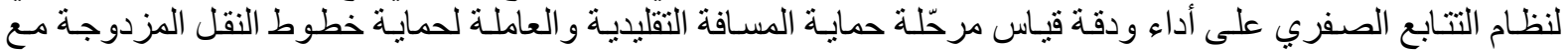


وبدون تأثير مقاو مة العطل وتحت ظروف تشغيلية ومو اقع عطل مختلفة. وقد أظهرت النتائج التي تم الحصول عليها من

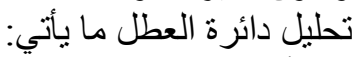

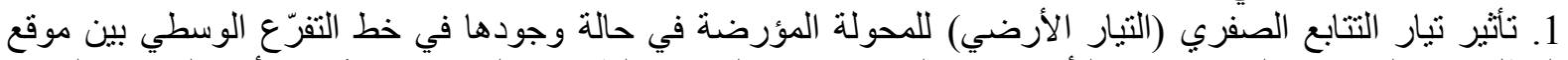

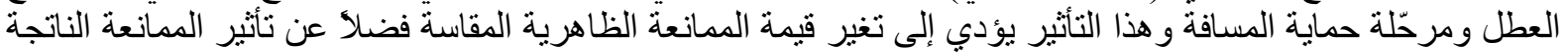

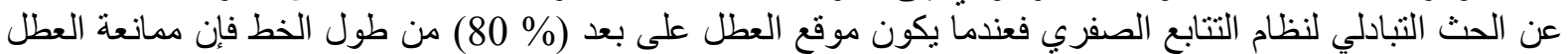
الفعلية تكون بحدود (16.705 2 (69.8)

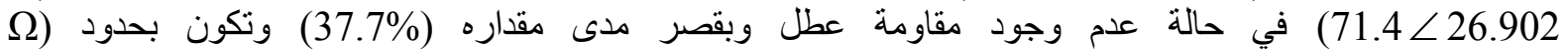

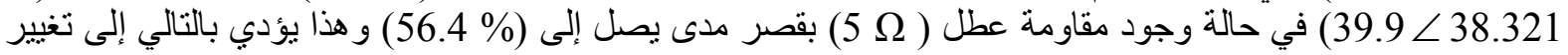
طول منطقة الحماية في كلا الحالتين.

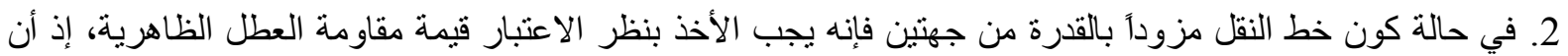

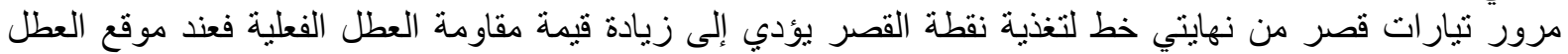

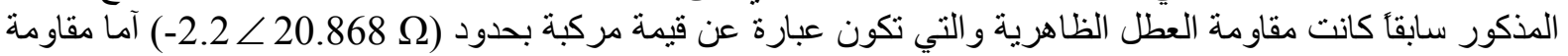

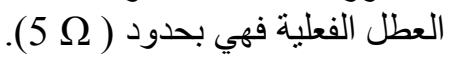

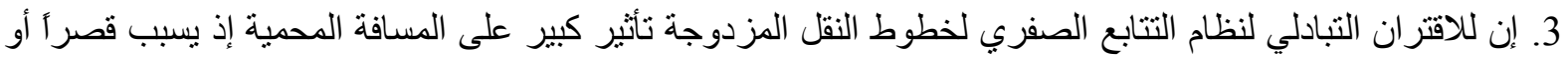

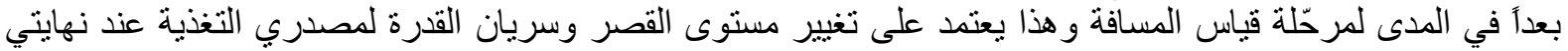

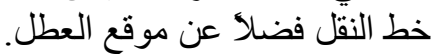

[1] Q. Gong, Y. Chen, C. Zhang and Z. Wang, “A Study of the Accurate Fault Location System for Transmission Line Using Multi-Terminal Signals ”, Proceeding Paper, IEEE PES Winter Meeting, Vol. 4, pp.2533-2538, 2000.

[2] T. Nagasawa, M. Abe, N. Otsuzuki, T. Emura, Y.Jikihara, M. Takeuchi, " Development of a New Fault Location Algorithm For Multi-Terminal Two Parallel Transmission Lines ”, IEEE Transactions on Power Delivery, Vol. 7, No. 3, pp. 1516-1532, July 1992.

[3] D.A. Tziouvaras, J.B. Roberts and G. Benmouyal, " New Multi-Ended Fault Location Design For Two or Three Terminal Lines ", IEEE Developments in Power System Protection, Conference Publication No.479, pp. 395-398, 2001.

[4] Y.Q. Xia, A.K. David and K.K. Li, "High-Resistance Faults on a Multi- Terminal Line: Analysis, Simulated Studies and an Adaptive Distance Relaying Scheme ", IEEE Transactions on Power Delivery, Vol.9, no 1, pp. 492- 500, January 1994.

[5] ALSTOM, " Protection and Automation Net Work ", Guide, Alstom T\&D Energy Automation and Information, Peter Rush, Levauios - Perret France, 2002.

[6] Gerhard Ziegler, “Numerical Distance Protection, Principles and Application ”, Siemens, Erlangen, Publics -MCD-Verl. 2008.

[7] G. E. Alexander and J. G. Andrichak, "Application of Phase and Ground Distance Relay to Three terminal Lines ", GE protection \& control, Malven, PA, 1994.

[8] M. F. AL-Kababjie, D. A. AL-Na'ma, H. I. Zynal, and N. Sheet, "Evaluation of Distance protection Devices High Voltage North Net Work", The University of Mosul, Presented to I.N.R.G., August 2001.

د. عبد الغني عبد الرزاق و السيد حسن حجي خديدة،“ دراسة نأثير نقاط التأريض على مستويات تيار [9] القصر لثبكة المنطقة الثمالية 132 kV “، جامعة الموصل, الثركة العامة لإنتاج الطاقة الكهربائية للمنطقة الثمالية, أيلول, 2001.

$$
\text { تم اجراء البحث في كلية ألهندسة = جامعة ألموصل }
$$

